\title{
Análisis de una Cohorte de Pacientes con ACV del Joven. Hospital de Clínicas, Montevideo.
}

\author{
Analysis of a cohort of patients with young man's stroke. \\ Hospital of Clinics, Montevideo. \\ Análise de uma coorte de pacientes com AVC do jovem. \\ Hospital de Clínicas, Montevidéu.
}

Juan Higgie Neurólogo. Asistente de Neurología.

Luis Urban Neurólogo.

H. Johen Hackembruch Asistente de Neurología Neurofisiología clínica.

Andrés Gaye Profesor Agregado de Neurología.
Resumen: Introducción. El ataque cerebro-vascular (ACV) es un importante problema de salud pública. Se define ACV en el joven a aquel que se presenta entre los 15-45 años. El objetivo primario de este trabajo es describir las características epidemiológicas, clínicas, imagenológicas, etiopatogénicas y terapéuticas de los pacientes con ACV en el joven. Materiales y Métodos. Se trata de un estudio retrospectivo y descriptivo, acerca de una cohorte de pacientes que presentaron ACV en el joven asistidos en el Hospital de Clínicas de Montevideo en el período comprendido entre junio de 2007 y junio de 2014. Resultados. Se incluyeron 50 pacientes que representaron el 5,6 \% de todos los ACV en el período comprendido en el estudio. La media de edad fue de 33 años. En cuanto a la naturaleza, el $64 \%$ fueron isquémicos y el $36 \%$ hemorrágicos. Según la clasificación TOAST, la etiología más frecuente fue la criptogenética (34\%). Respecto a la etiología de las hemorragias intracerebrales la mitad correspondió a malformaciones vasculares. Discusión. El porcentaje de ACV del joven fue algo menor que el descrito en otras comunicaciones. Comparado con el ACV en todas las edades, la hemorragia cerebral aumenta su proporción, si bien la naturaleza isquémica sigue predominando. La causa más frecuente de ACV isquémico del joven fue la criptogenética, seguida de la cardioembolia y las causas inhabituales. Las disecciones arteriales igualaron en prevalencia a la ateromatosis de grandes vasos. Este hecho es similar a lo descrito por la mayoría de los autores. Si bien la etiología más prevalente de hemorragia parenquimatosa fueron las malformaciones vasculares, la cantidad de hematomas hipertensivos no fue despreciable a estas edades.

Palabras clave: Unidad ACV, ACV, joven.

Abstract: Introduction. Stroke is an important public health issue. Stroke in the young is defined as the one who presents between 15 and 45 years old. The primary objective of this study is to describe the epidemiological, clinical, imaging, etiopathogenic and therapeutic characteristics of stroke in young patients. Materials and Methods. This was a retrospective and descriptive study of a cohort of patients who presented with stroke in the young assisted at the Hospital de Clínicas in Montevideo in the period between June 2007 and June 2014. Results. We have included fifty patients which accounted for $5.6 \%$ of all stroke in the period covered by the study. The mean age was 33 yo. As for nature, $64 \%$ were ischemic and $36 \%$ hemorrhagic. According to the TOAST classification, the most common etiology was cryptogenetic (34\%). Regarding the etiology of intracerebral hemorrhages, half corresponded to vascular malformations. Discussion. The proportion of stroke in young people was somewhat lower than that described in other communications. Compared with stroke in all ages, cerebral hemorrhage increases its proportion, although the ischemic nature continues to predominate. The most common cause of ischemic stroke was cryptogenetic, followed by cardioembolism and unusual causes. Arterial dissections matched the prevalence of atheromatosis of large vessels. This fact is similar to that described by most authors. Although the most prevalent etiology of parenchymal hemorrhage were vascular malformations, the number of hypertensive hematomas was not negligible at these ages. 
Keywords: Stroke unit, stroke, young patients

Resumo: Introdução. O ataque cerebrovascular (AVC) é um importante problema de saúde pública. A ACV é definida no jovem que aparece entre 15 a 45 anos. O objetivo primário deste trabalho é descrever as características epidemiológicas, clínicas, de imagem, etiopatogênicas e terapêuticas de pacientes com AVC em jovens. Materiais e métodos. Trata-se de um estudo retrospectivo e descritivo sobre uma coorte de pacientes que se apresentaram com AVC no jovem atendido no Hospital de Clínicas de Montevidéu no período de junho de 2007 a junho de 2014. Resultados. Cinquenta pacientes foram incluídos, representando $5,6 \%$ de todos os casos de AVC no período coberto pelo estudo. A idade média foi de 33 anos. Em relação à natureza, $64 \%$ eram isquêmicos e $36 \%$ eram hemorrágicos. Segundo a classificação TOAST, a etiologia mais freqüente foi a criptogenética (34\%). Em relação à etiologia das hemorragias intracerebrais, metade correspondeu a malformações vasculares. Discussão. A porcentagem do derrame do jovem foi um pouco menor do que a descrita em outras comunicações. Em comparação com o AVC em todas as idades, a hemorragia cerebral aumenta sua proporção, embora a natureza isquêmica continue a predominar. A causa mais freqüente de acidente vascular cerebral isquêmico do jovem foi criptogenética, seguida por cardioembolismo e causas incomuns. Dissecções arteriais igualaram a prevalência de ateromatose de grandes vasos. Este fato é semelhante ao descrito pela maioria dos autores. Embora a etiologia mais prevalente da hemorragia parenquimatosa tenha sido a malformação vascular, o número de hematomas hipertensivos não foi desprezível nessas idades.

Palavras-chave: Unidade ACV, ACV, jovem

Recibido: 15/03/2018 - Aceptado: 22/04/2018

Unidad de ACV del Instituto de Neurología. Hospital de Clínicas "Dr. Manuel Quintela." Facultad de Medicina. Universidad de la República. Montevideo - Uruguay. 


\section{Introducción}

El ataque cerebro-vascular (ACV) es un importante problema de salud pública tanto a nivel local como mundial. En Uruguay, su incidencia se ha estimado en 181 casos cada 100.000 habitantes/año (lo que representa unos 5500 a 6000 ACV por año) (1) y una tasa de mortalidad bruta de 93 casos cada 100.000 habitantes/año ${ }^{(2)}$, siendo la segunda causa de muerte detrás del infarto agudo de miocardio y la primera en mujeres mayores de 65 años (Ministerio Salud Pública). El mismo genera una significativa carga de enfermedad, por años de vida saludables perdidos por discapacidad y muerte prematura, siendo esto especialmente dramático en el caso del ACV en el joven debido a la edad en la cual se presenta ${ }^{(3)}$.

Se define ACV en el joven a aquel que se presenta entre los 15-45 años, si bien existen definiciones que amplían el rango superior hasta los 50 años. Esta ampliación suele generar inclusión de pacientes con patologías típicas de los más añosos (ateromatosis, enfermedad de pequeño vaso y otras), por lo que el límite superior de 45 años es el más utilizado.

Habitualmente se considera al ACV como una patología de las personas adultas. Si bien es cierto que la incidencia del mismo está fuertemente correlacionada con la edad, el 8-12 \% ocurren en pacientes menores de 45 años ${ }^{(4-7)}$. La incidencia de ACV en este grupo etario va en aumento, aunque las razones no están del todo claras ${ }^{(3,8)}$. El conocer dichas razones y tratar de prevenirlas es de fundamental importancia dado que los pacientes con ACV en el joven tienen un riesgo de muerte temprana aumentado en comparación con la población general de igual edad ${ }^{(6,8,9)}$ y generan una carga muy elevada de años de vida asociados a discapacidad.

El estudio de las causas de ACV en el joven también es de sumo interés debido a las potenciales diferencias etiológicas del ACV con respecto a las personas mayores ${ }^{(6)}$. La enfermedad de pequeño vaso, la aterosclerosis de grandes vasos y la fibrilación auricular, que son responsables de la mayoría de los casos de ACV isquémico en pacientes de edad avanzada, son menos comunes en los adultos jóvenes ${ }^{(10-12)}$. Asimismo, son más frecuentes algunas etiologías como la disección arterial, estados protrombóticos, cardiopatías embolígenas diferentes a la fibrilación auricular (FA), entre otras, que si bien son responsable de la etiología del ACV en casos aislados, dado el pronóstico que conllevan y el eventual cambio en la conducta terapéutica, hacen imprescindible su búsqueda mediante un abordaje más exhaustivo ${ }^{(5,6,10-12)}$.

Si bien es cierto que en este subtipo de ACV las etiologías son más diversas que en el ACV de los añosos, en los mayores de 35 años las etiologías se van asemejando a las del ACV de la población general ${ }^{(6,11,13)}$. Así mismo las hemorragias cerebrales presentan más variadas y distintas etiologías en este grupo y aumenta su proporción respecto a los ACV isquémicos ${ }^{(5,6)}$.

A pesar de una minuciosa búsqueda etiológica, un 30-35\% aproximadamente de los casos de ACV isquémicos en el joven quedan sin etiología aclarada (ACV indeterminados a pesar de estudio completo o criptogénicos) ${ }^{(6,13,14)}$. Por lo tanto el principal reto para el neurólogo que se enfrenta ante un paciente con ACV en el joven es identificar su causa para lograr un correcto manejo terapéutico y la prevención secundaria adecuada del mismo ${ }^{(5,6,11)}$.

\section{Objetivos}

El objetivo primario de este trabajo es describir las características epidemiológicas, clínicas, imagenológicas, etiopatogénicas y terapéuticas de los pacientes con ACV en el joven.

En cuanto a los objetivos secundarios interesa evaluar:

1) Las causas de cardioembolia encontradas en los estudios solicitados para valoración de los ACV isquémicos en estos pacientes.

2) Los hallazgos patológicos encontrados a nivel de la vasculatura intracraneana.

3) Las causas de etiopatogenia inhabitual. En el caso de los ACV hemorrágicos valoraremos:

4) Las distintas malformaciones vasculares causantes de hematomas intracraneanos en la población.

En cuanto a las medidas terapéuticas interesa conocer:

5) Las causas de no realización de trombolisis intravenosa en pacientes con ACV isquémico del joven que llegaron dentro de la ventana temporal para su realización.

6) Por último, se describe la variabilidad estacional de la presentación de los eventos cerebro-vasculares. 


\section{Materiales y Métodos}

Se trata de un estudio retrospectivo y descriptivo, acerca de una cohorte de pacientes que presentaron ACV en el joven asistidos en el Hospital de Clínicas de Montevideo en el período comprendido entre junio de 2007 y junio de 2014.

La fuente de recolección de los datos fueron los protocolos de todos los pacientes con ACV en la base de datos del registro de la Unidad de ACV (UACV) de dicho Hospital en el período mencionado. Se seleccionaron los ACV que ocurrieron en los pacientes jóvenes y los datos obtenidos se recogieron en una ficha realizada específicamente para este estudio.

Se definió como ACV en el joven aquel que ocurrió en pacientes entre 15 y 45 años.

Se excluyeron los pacientes con infarto venoso y hemorragia subaracnoidea.

Para la descripción de las variables cualitativas se utilizaron porcentajes y para las variables cuantitativas valores de media, mediana y desvío estándar. Se compararon variables cuantitativas y cualitativas utilizando los estadísticos Chi cuadrado y prueba T para muestras no relacionadas. Se tomó un valor de significación de 0,05.

\section{Resultados}

Se incluyeron 50 pacientes con ACV del joven que representaron el 5,6 \% de todos los ACV en el período comprendido en el estudio.

La media de edad fue de 33 años $(D E \pm 9.3)$ con un rango entre 16 y 45 años. Del total de pacientes, 26 de ellos fueron $\leq 35$ años (media de 25 años $D E \pm 6$ ) y 24 casos tuvieron $>35$ años (media de 41 años $\mathrm{DE} \pm 2$ ).

La distribución por sexo fue de un $52 \%$ para mujeres y un $48 \%$ para hombres.

Teniendo en cuenta la naturaleza del ACV, 32 de ellos (64\%) fueron isquémicos y 18 casos (36\%) fueron hemorrágicos.

No se observaron diferencias significativas en el sexo en relación al subtipo de ACV isquémico o hemorrágico (Chi2. $p=0.83$ )

Sí observamos una diferencia de edad, con una media de edad menor (media $27 \mathrm{DE} \pm 10$ ) en los pacientes con ACV hemorrágico que en los pacientes con ACV isquémico (media $36 \mathrm{DE} \pm 7$ ) la cual fue significativa. (Prueba T. $p=0,002$ ) (Gráfico 1).

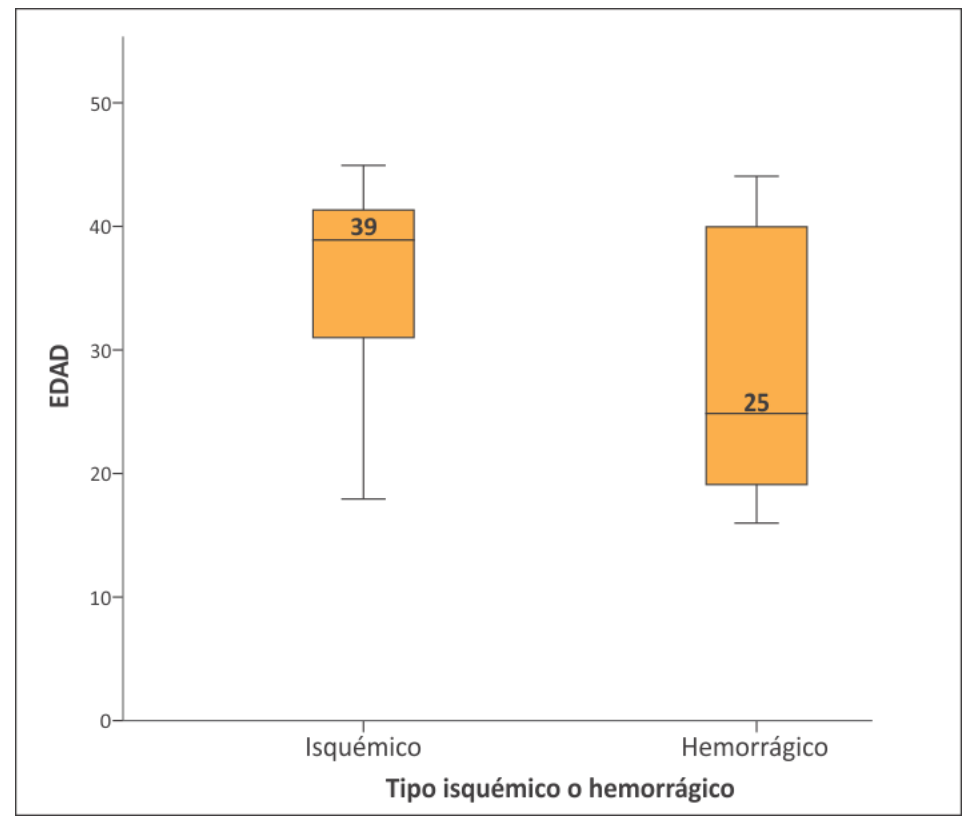


No encontramos diferencias significativas entre la escala de NIHSS al ingreso y el subtipo de ACV (Gráfico 2).

Gráfico 2: Box plot que muestra la mediana de NIHSS al ingreso de los ACV isquémicos y los hematomas.

Tabla 1: Cuadro clínico según naturaleza

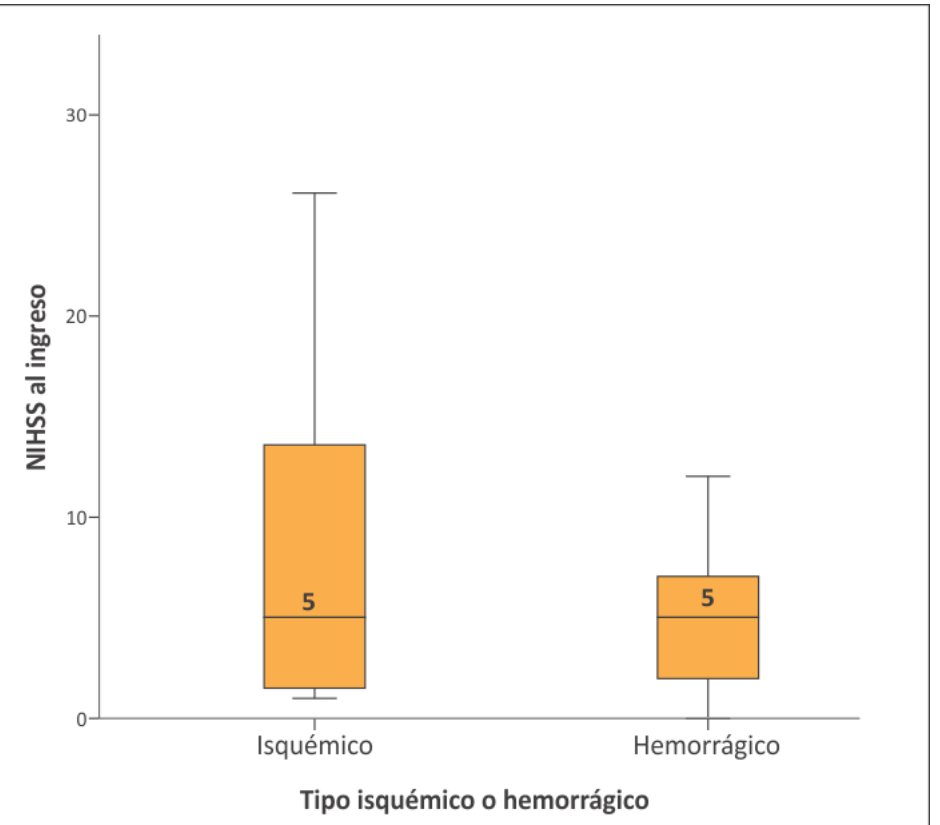

La distribución de ACV según la estación del año en que ocurrieron se muestra en el gráfico 3.

Gráfico 3: Distribución del ACV según estación del año.

Las características clínicas de presentación de los ACV isquémicos y hemorrágicos y así como sus antecedentes personales se representan en las tablas 1 y 2.

\begin{tabular}{|l|l|}
\hline \multicolumn{1}{|c|}{ Clinica ACV isquémicos } & \multicolumn{1}{c|}{ Clínica ACV hemorrágicos } \\
\hline Síndrome piramidal $77.4 \%$ & Síndrome piramidal $55.5 \%$ \\
\hline Afasia $38.7 \%$ Agnosia $9.6 \%$ & Hipertensión endocraneana $50 \%$ \\
\hline Síndrome sensitivo deficitario $32.2 \%$ & Síndrome vestibular $22.2 \%$ \\
\hline Síndrome sensorial visual def $19.3 \%$ & Trastornos oculomotores $16.6 \%$ \\
\hline Hipertensión endocraneana $19.3 \%$ & Afasia $16.6 \%$ \\
\hline Síndrome cerebeloso hemisférico $9.6 \%$ & Crisis epilépticas $16.6 \%$ \\
\hline Síndrome cerebeloso vermiano $6.4 \%$ & Síndrome cerebeloso hemisférico $11.1 \%$ \\
\hline Trastornos oculomotores $6.4 \%$ & Síndrome cerebeloso vermiano $11.1 \%$ \\
\hline Crisis epilépticas $6.4 \%$ & Síndrome sensitivo deficitario $11.1 \%$ \\
\hline Síndrome alterno $6.4 \%$ & Síndrome alterno $5.5 \%$ \\
\hline
\end{tabular}

Tabla 2: Antecedentes personales de los pacientes según naturalez

\begin{tabular}{|l|l|}
\hline \multicolumn{1}{|c|}{ Antecedentes personales ACV isquémicos } & \multicolumn{1}{|c|}{ Antecedentes personales ACV hemorrágicos } \\
\hline Tabaquismo $56.2 \%$ & Hipertensión arterial $27.7 \%$ \\
\hline Hipertensión arterial $31.2 \%$ & Tabaquismo $27.7 \%$ \\
\hline Alcoholismo $18.7 \%$ & ACV previo $11.1 \%$ \\
\hline AlT previo $15.6 \%$ & AlT previo $5.5 \%$ \\
\hline ACV previo $9.3 \%$ & Dislipemia $5.5 \%$ \\
\hline Dislipemia $6.2 \%$ & Arritmia $5.5 \%$ \\
\hline Diabetes Mellitus $3.1 \%$ & Alcoholismo $5.5 \%$ \\
\hline Arritmia $3.1 \%$ & Diabetes Mellitus $0 \%$ \\
\hline Infarto de miocardio $0 \%$ & \\
\hline
\end{tabular}

De la presentación clínica se destaca una diferencia significativa en la presencia de hipertensión endocraneana entre los hematomas (50\%) versus los ACV isquémicos (19.3\%). (Chi2. Fisher. $\mathrm{p}=0,02$ ).

En cambio la presencia de crisis epilépticas, si bien fue más frecuente en los ACV hemorrágicos (16,6\%), la diferencia entre uno y otro grupo no fue significativa (Chi2. $p=0,32$ ). 
En cuanto a los estudios para valorar la etiopatogenia, a todos los pacientes con ACV isquémico se les realizó un electrocardiograma, de los cuales el 3,4\% evidenció un alto riesgo de cardioembolia, al mostrar una fibrilación auricular; mientras que el $96.6 \%$ no asoció elementos de riesgo electrocardiográfico. Contamos con ecocardiograma en 29 de los 32 pacientes con ACV isquémico $(90,6 \%)$, de los cuales 13 tienen, además ecocardiograma transesofágico. El estudio ecocardiográfico evidenció un alto riesgo de cardioembolia en el 14,8 \% de los pacientes. El mismo porcentaje de estudios mostró un riesgo moderado, mientras que en el $70,3 \%$ de los casos se obtuvo un ecocardiograma sin elementos de riesgo para cardioembolia.

Los ecocardiogramas mencionados como de alto riesgo evidenciaron: prótesis valvular mecánica en 2 casos, trombo en la aurícula izquierda en 1 caso y elementos sugestivos de endocarditis infecciosa en el restante. Dentro de los ecocardiogramas de mediano riesgo se encuentran los siguientes hallazgos: signos de insuficiencia cardíaca congestiva en uno de los pacientes, 2 de ellos con foramen oval permeable (FOP) y 1 uno con comunicación interauricular $(\mathrm{CIA})$.

El ecodoppler de vasos de cuello mostró compromiso de la circulación anterior en el 10,7\% de los casos, compromiso de la circulación posterior en el 3,6\% y no mostró compromiso en el $85,7 \%$ de los pacientes. Para la misma se tomó en cuenta una estenosis significativa $>50 \%$.

La clasificación clínica de Oxford y la etiopatogénica de TOAST para los ACV isquémicos se representan en los gráficos 4 y 5 .
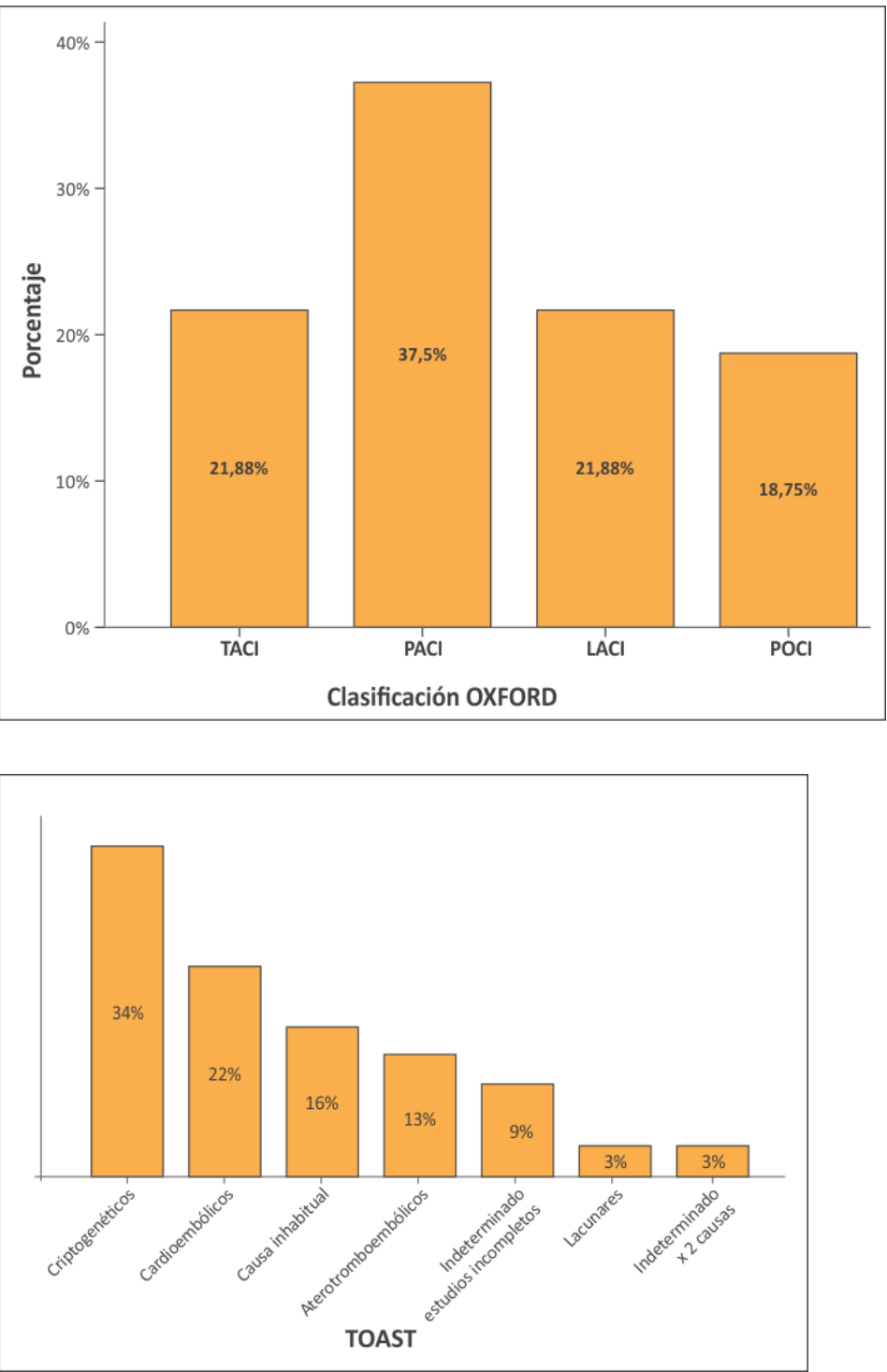
De los pacientes clasificados como de etiología indeterminada, 3 de ellos tuvieron estudios incompletos, en 1 caso se evidenció más de una causa posible y 11 presentaron estudios completos negativos (verdaderos criptogénicos).

En cuanto a la topografía por neuroimagen, el 78,5\% mostró infarto de la circulación anterior mientras que el $21,5 \%$ fue de la circulación posterior.

De los 32 pacientes que presentaron un ACV de naturaleza isquémica, 6 de ellos llegaron en el plazo de tiempo para la administración de trombolítico intravenoso. El mismo se pudo realizar en 2 de los pacientes (33,3\% de los que llegaron en plazo, 6,25\% del total de los ACV isquémicos), mientras que en 4 de ellos no se logró realizar por los siguientes criterios de exclusión: sangrado activo significativo en un caso, signos tomográficos de infarto extenso en 2 casos y NIHSS mayor de 25 en el caso restante.

En cuanto a los ACV hemorrágicos, el 33\% correspondieron a hematomas vinculados con HTA, un $50 \%$ se debió a malformaciones vasculares (angioma cavernoso en 3 pacientes, malformación A-V en 5 casos y fistula pial en un paciente), mientras que el $17 \%$ fue de causa indeterminada ( 2 pacientes por estudios incompletos y uno por estudios completos negativos)

La topografía de los hematomas y el score de hemorragia (predictor de mortalidad para la hemorragia intraparenquimatosa) se observan en los gráficos 6 y 7 .
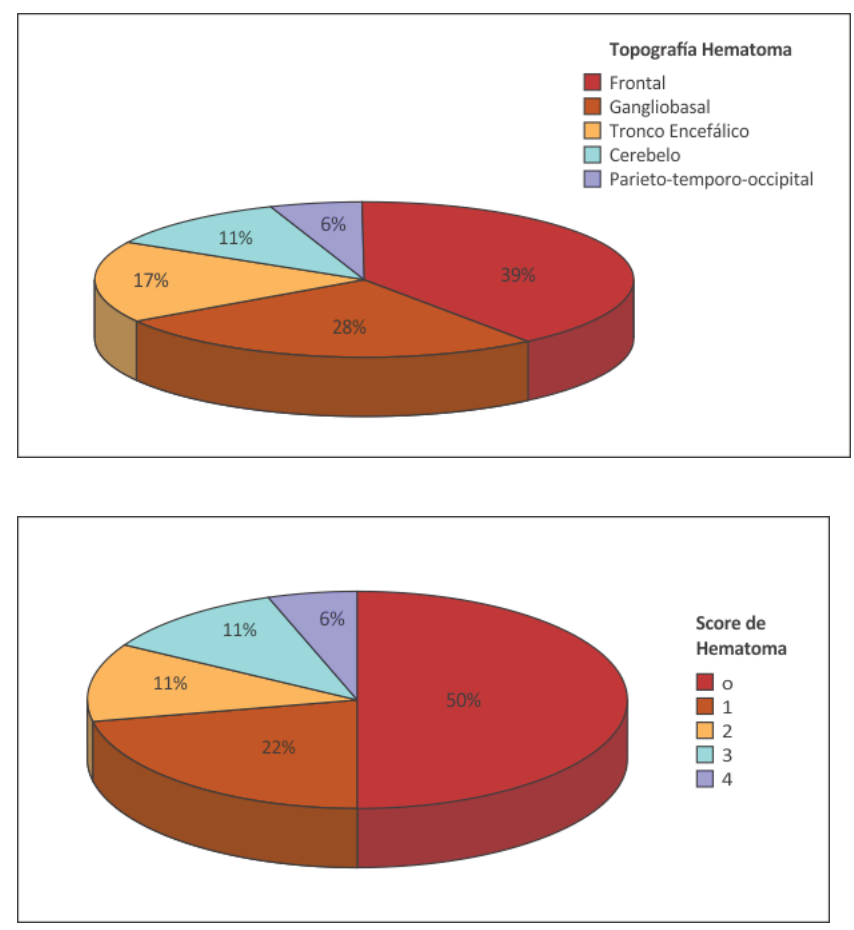

\section{Discusión}

Los ACV del joven representaron el 5,6 \% del total de los ACV en su conjunto, cifra ligeramente menor a la referida en los trabajos internacionales, la cual ronda el $10 \%$, y algo inferior a la reportada en un trabajo nacional, estimada en $8 \%{ }^{(15)}$.

No hubo diferencias significativas entre la proporción de mujeres y hombres, 52 y $48 \%$ respectivamente. En algunos trabajos se habla de una preponderancia del sexo femenino en este grupo etario debido a factores hormonales o del embarazo y puerperio. Este hecho no se dio en nuestra serie, quizás por la baja frecuencia de síndromes protrombóticos y de ACV peri embarazo detectado en el estudio.

En cuanto a la naturaleza del ACV, los hematomas representaron un porcentaje de $36 \%$, proporción mayor a la habitualmente encontrada en los ACV de cualquier edad donde representan entre un 15 y $20 \%$. Este porcentaje mayor de hemorragia cerebral sigue la tendencia de los datos publicados, donde el hematoma da cuenta de valores cercanos a $50 \%$ de los ACV del joven ${ }^{(3,5,6)}$ y se relaciona con el mayor peso relativo de las malformaciones vasculares en este grupo respecto a los sujetos más añosos. En la serie nacional comunicada en ANFAMED ${ }^{(15)}$, 
el $60 \%$ de los ACV del joven eran hemorragias cerebrales y predominaban las malformaciones vasculares.

Una posible explicación para el menor porcentaje de ACV hemorrágicos en nuestra serie respecto a otras es que se excluyeron las hemorragias subaracnoideas, pues en la fuente de obtención de datos utilizada, éstas no están registradas.

En cuanto a la presentación clínica de los pacientes portadores de hematoma se encontró que los 2 síndromes más frecuentes fueron el piramidal y el de hipertensión endocraneana, con una frecuencia de 55,5 y $50 \%$ respectivamente.

En los ACV isquémicos el síndrome clínico más frecuente fue el síndrome piramidal con una frecuencia del $77,4 \%$, seguido en forma más alejada por el trastorno del lenguaje con un $38,7 \%$. La hipertensión endocraneana fue claramente más frecuente en el grupo de pacientes con hematomas que en los pacientes con infarto cerebral (50\% vs $19,3 \%$ ), lo cual fue estadísticamente significativo. De todas formas no fue un síntoma uniformemente presente en los ACV hemorrágicos, confirmando su baja sensibilidad para el diagnóstico clínico de naturaleza del evento.

Las crisis epilépticas también fueron más frecuentes entre los pacientes con hematomas (16,6\% vs $6,4 \%)$, con una diferencia no significativa. De todas formas la frecuencia global de las mismas es baja, reafirmando la no necesidad de uso profiláctico de drogas antiepilépticas en la gran mayoría de estos pacientes.

Respecto a los antecedentes personales, en los pacientes con hematoma se destaca un bajo porcentaje relativo de hipertensión arterial $(27,7 \%)$ coincidiendo con los datos de la literatura ${ }^{(15)}$. Esta es la explicación de por qué el hematoma hipertensivo no fue la causa más frecuente como se observa en los sujetos añosos donde la prevalencia de HTA es mucho más elevada.

En los pacientes con ACV isquémico vimos un bajo porcentaje de factores de riesgo cardiovascular clásicos como la diabetes y la dislipemia; mientras que el tabaquismo y la hipertensión arterial fueron muy prevalentes (HTA 31,2\%, DM 3,1\%, dislipemia 6,2\%, tabaquismo $56,2 \%)^{(3,8,11,13)}$, reafirmando que en los pacientes jóvenes los FRCV clásicos tienen también una gran importancia, sobre todo en los mayores a 35 años.

En cuanto a la estacionalidad de la ocurrencia de los ACV se observó una mayor incidencia en la estación de otoño en ambos grupos, el de tipo isquémico en un $40 \%$ y el hemorrágico en un $44 \%$ del total respectivamente; siendo el verano la estación de menor incidencia (ACV isquémico: $15 \%$ y ACV hemorrágico: $11 \%$ ). Esto podría deberse a un aumento en las cifras de presión arterial en los meses más fríos del año, hecho que ya ha sido comunicado.

Si analizamos la clasificación clínica de subtipos de ACV isquémico de Oxford, se encuentran cifras similares a las encontradas en el trabajo original de dicha clasificación, siendo los más frecuentes los PACI (infartos parciales de circulación anterior). El hecho de que el ACV isquémico tiende a ser globalmente menos grave que en el sujeto añoso también ha sido descrito.

Dentro de los mecanismos etiopatogénicos del Infarto Cerebral según clasificación TOAST, la etiología más frecuente fue la criptogenética con una frecuencia del 34\%. La segunda correspondió a la cardioembolia en un $22 \%$ y en tercer lugar a la causa inhabitual con un $16 \%$. Dentro de las causas inhabituales se detectaron 2 disecciones arteriales de circulación anterior, 1 disección de circulación posterior, 1 vasculitis y una crisis vasooclusiva en un paciente con anemia depranocítica. La disección arterial fue por tanto la causa inhabitual más frecuente (sobre todo en el subgrupo menor de 36 años), siendo la etiología responsable en el $9 \%$ de todos los casos, similar a lo que reportan la mayoría de las series ${ }^{(7,11)}$.

El mecanismo aterotrombótico fue responsable del $9 \%$ de los ACV isquémicos. El resto de los casos fueron indeterminados por 2 o más causas posibles o indeterminados por estudios incompletos.

Los datos acerca de la etiología son similares a los publicados en la literatura. Sin embargo, existen variaciones en la proporción de pacientes en los distintos subgrupos etiológicos de los trabajos publicados. La explicación es la diferencia en los criterios operacionales que definen a cada subgrupo etiológico, así como a la extensión de estudios paraclínicos solicitados en la valoración del ACV en los distintos centros.

El bajo porcentaje de mecanismo aterotrombotico: $9 \%$, coincide con los datos comunicados, dado que en esta franja etaria la ateromatosis es menos frecuente ${ }^{(7,14)}$. 
La cardioembolia es responsable de entre el 20-30\% de los ACV en el joven en la literatura internacional. Encontramos un similar porcentaje de $22 \%$ en nuestro trabajo, siendo el FOP una de las principales causas posibles de cardioembolia, tal y como está descrito en dichos estudios $^{(7,11)}$. De todos modos, el rol causal del FOP en los infartos cerebrales es controvertido, a excepción de cuando coexiste con una trombosis venosa profunda y embolia paradojal.

La frecuencia de mecanismo cardioembólico en los menores de 36 años comparada con los mayores de 35 años fue similar, sin embargo el mecanismo lacunar fue más frecuente en los menores de 36 años; siendo esto inverso a lo descrito en la literatura, seguramente por el bajo número de pacientes que tenemos en ambos subgrupos.

Si analizamos los casos desde la disponibilidad del tratamiento fibrinolítico en febrero de 2010 , el mismo fue realizado en el $9 \%$ de los pacientes con ACV del joven. En un $19 \%$ de los casos no se realizó rtpa a pesar de llegar los pacientes en plazo, ya sea por sangrado activo significativo, NIHSS mayor a 25 o por signos tomográficos de infarto extenso. No existieron diferencias significativas en el porcentaje de pacientes trombolizados en el subgrupo de ACV joven versus la población total de ACV.

En cuanto a la topografía del ACV por neuroimagen, 4 de cada 5 correspondió a la circulación anterior como es esperable según las referencias analizadas.

En cuanto a la etiología de las hemorragias intracerebrales la mitad correspondió a malformaciones vasculares, lo que concuerda con otros estudios ${ }^{(16)}$. Dentro de ellas la más frecuente fue la MAV, seguida del angioma cavernoso. En cuanto a la etiología hipertensiva, la misma solo se presentó en la tercera parte de los pacientes como es esperable en este subtipo de ACV. Sobre el uso de drogas, la ausencia de pacientes con hemorragia secundaria a esta etiología, podría ser explicada por la falta de solicitud de screening para las mismas.

Acerca del score pronóstico de mortalidad en hemorragia cerebral, la mitad de los pacientes se presentó con un score de 0 el cual se corresponde al mejor pronóstico, seguido por el score de 1 con un $20 \%$ aproximadamente, por lo que el $70 \%$ de los pacientes tenían, a priori, un buen pronóstico vital.

A diferencia del ACV hemorrágico en todos los grupos etarios, en este subgrupo de jóvenes, la topografía más habitual no fue la ganglio-basal. La mayor frecuencia correspondió a la topografía lobar y dentro de ellas a la región frontal, hecho vinculado al menor número de casos con etiología hipertensiva y a la mayor frecuencia de malformaciones vasculares, las cuales tienden a localizarse en topografías superficiales; estando estos datos en concordancia con los estudios publicados y analizados ${ }^{(16)}$.

\section{Conclusiones}

1) El ACV en el joven correspondió al 5,6\% de todos los ACV.

2) El $64 \%$ de los ACV fueron isquémicos y el $36 \%$ fueron de naturaleza hemorrágica.

3) En cuanto a la etiología de los isquémicos, la más frecuente fue la criptogénica (34\%), seguida de la cardioembolia, la etiología inhabitual, la aterotromboembolia y por último la patología de pequeño vaso.

4) La etiología más frecuente de hematoma intraparenquimatoso fueron las malformaciones vasculares encefálicas.

5) Los pacientes con ACV isquémico tuvieron una media de edad mayor (36 años) a los pacientes con hematoma (27 años), siendo dicha diferencia significativa. 


\section{Bibliografía}

1- Hochmann B, Coelho J, Segura J, Galli M, Ketzoian C, Pebet M. Incidencia del accidente cerebrovascular en la ciudad de Rivera, Uruguay. Rev Neurol 2006;43 : $78-83$.

2- Hackembruch H, Perna A, Ketzoian C. Mortalidad por ACV en el Uruguay. En: XX World Congress of Neurology. Marruecos; 2011

3- Singhal AB, Biller J, Elkind MS, Fullerton HJ, Jauch EC, Kittner SJ, et al. Recognition and management of stroke in young adults and adolescents. Neurology. 2013;81(12): 1089-1097.

4- Nagaraja N, Bhattacharya P, Norris G, Coplin W, Narayanan S, Xavier A, Rajamani K. Arrival by ambulance is associated with acute stroke intervention in young adults. J Neurol Sci. 2012 May 15;316(1-2):168-9.

5- Martin, PJ, Enevoldson TP, Humphrey PR. Causes of ischaemic stroke in the young. Postgrad Med J. 1997 Jan;73(855):8-16

6- Varona F, Guerra JM, Bermejo F. Ictus en el adulto joven. Med clín. 2004; 122(2): 70-74.

7- Ji R, Schwamm LH, Pervez MA, Singhal AB. Ischemic stroke and transient ischemic attack in young adults: risk factors, diagnostic yield, neuroimaging, and thrombolysis. JAMA neurol. 2013; 70(1): 51-57.

8- Mackey J. Evaluation and management of Stroke in Young Adults. Continuum (Minneap Minn). 2014 Apr;20 (2 Cerebrovascular Disease):352-69. doi: 10.1212/01.CON.0000446106.74796.e9.

9- Sultan S, Elkind MS. The growing problem of stroke among young adults. Curr Cardiol Rep. 2013 Dec;15(12):421. doi: 10.1007/s11886-013-0421-z.

10- Larrue V, Berhoune N, Massabuau P, Calviere L, Raposo N, Viguier A, et al. Etiologic investigation of ischemic stroke in young adults. Neurology. 2011; 76(23): 1983-1988.

11- Ferro Ferro JM, Massaro AR, Mas JL. Aetiological diagnosis of ischaemic stroke in young adults. Lancet Neurol. 2010; 9(11): 1085-1096.

12- Kuruvilla A, Bhattacharya P, Rajamani K, Chaturvedi S. Factors associated with misdiagnosis of acute stroke in young adults. J Stroke Cerebrovasc Dis.2011; 20(6): 523-527.

13- Renna R, Pilato F, Profice P, Della Marca G, Broccolini A, Morosetti R, et al. Risk factor and etiology analysis of ischemic stroke in young adult patients. J Stroke Cerebrovasc Dis. 2014;23(3): e221-e227.

14- Yamamoto, FI. Ischemic stroke in young adults: an overview of etiological aspects. Arq Neuropsiquiatr. 2012;70(6):462-6.

15- Gaye Saavedra A, Brunet F, Albisu S, Higgie J, Preve F, Camejo C, et al. Ataque Cerebrovascular en jóvenes en la Unidad de ACV del Hospital de Clínicas de Montevideo. An. Fac. Med. Univ. Republ. Montev. Urug. 2015;2 :70-76.

16- Ruíz-Sandoval JL, Cantú C, Barinagarrementeria F. Intracerebral Hemorrhage in Young People: analysis of risk factors, location, causes, and prognosis. Stroke. 1999;30:537-541. 